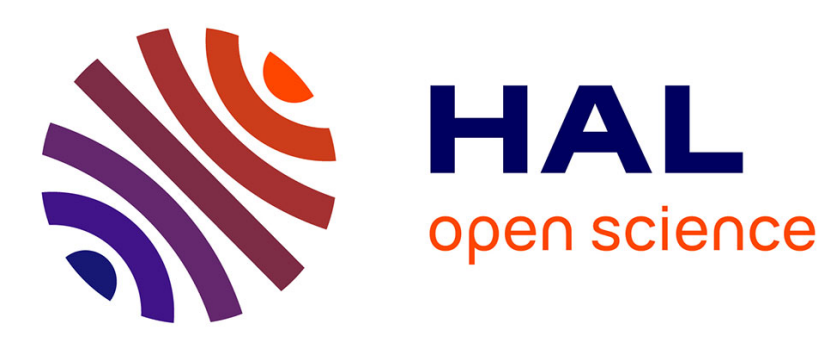

\title{
Congestion in Randomly Deployed Wireless Ad-Hoc and Sensor Networks
}

\author{
Alonso Silva, Patricio Reyes, Merouane Debbah
}

\section{To cite this version:}

Alonso Silva, Patricio Reyes, Merouane Debbah. Congestion in Randomly Deployed Wireless Ad-Hoc and Sensor Networks. [Research Report] RR-6854, INRIA. 2009. inria-00364370

\section{HAL Id: inria-00364370 \\ https://hal.inria.fr/inria-00364370}

Submitted on 26 Feb 2009

HAL is a multi-disciplinary open access archive for the deposit and dissemination of scientific research documents, whether they are published or not. The documents may come from teaching and research institutions in France or abroad, or from public or private research centers.
L'archive ouverte pluridisciplinaire HAL, est destinée au dépôt et à la diffusion de documents scientifiques de niveau recherche, publiés ou non, émanant des établissements d'enseignement et de recherche français ou étrangers, des laboratoires publics ou privés. 
INSTITUT NATIONAL DE RECHERCHE EN INFORMATIQUE ET EN AUTOMATIQUE

\section{Congestion in Randomly Deployed Wireless Ad-Hoc and Sensor Networks}

Alonso Silva - Patricio Reyes - Mérouane Debbah

\section{$\mathbf{N}^{\circ} 6854$}

February 2009

Thème COM

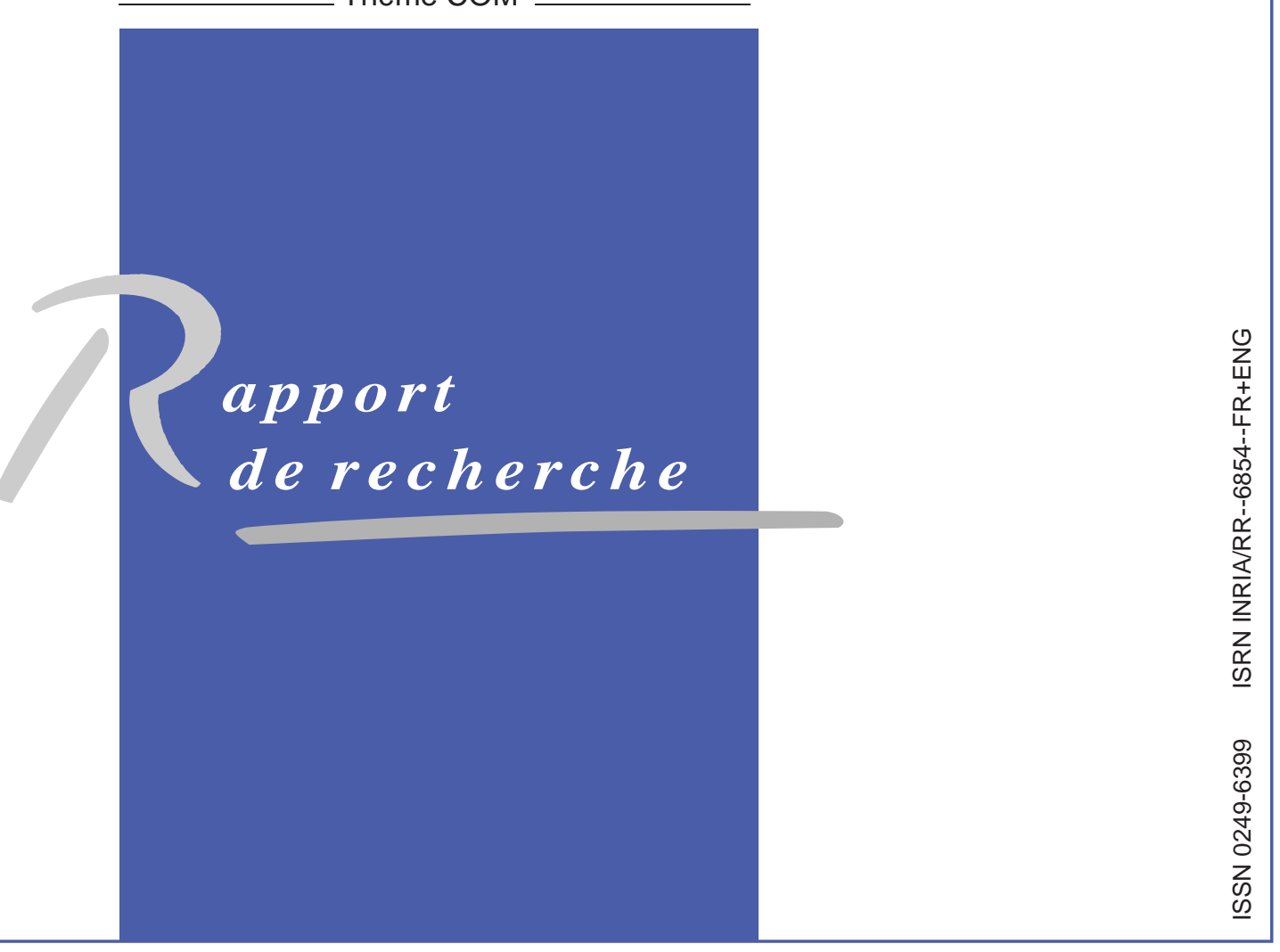





\title{
Congestion in Randomly Deployed Wireless Ad-Hoc and Sensor Networks
}

\author{
Alonso Silvat, Patricio Reyed, Mérouane Debbah \\ Thème COM — Systèmes communicants \\ Équipes-Projets Maestro \& Mascotte \\ Rapport de recherche $n^{\circ} 6854$ - February 2009 - 12 pages
}

\begin{abstract}
Congestion in wireless ad-hoc and sensor networks not only causes packet loss, and increases queueing delay, but also leads to unnecessary energy consumption. In a wireless ad-hoc and sensor network, two types of congestion can occur: node-level congestion, which is caused by buffer overflow in the node, or link-level congestion, when wireless channels are shared by several nodes and collisions occur when multiple active nodes try to seize the channel at the same time.

We study a measure of link-level congestion in a static wireless ad-hoc and sensor network randomly deployed over an area. The measure considered on this paper is the inverse of the greatest eigenvalue of the adjacency matrix of the random graph. This measure of congestion gives an approximation of the average quantity of wireless links of a certain length that a node have on the wireless ad-hoc and sensor network.

We review the results to find this measure of congestion in a Bernoulli random graph and we use tools from random graph theory and random matrix theory to extend this measure of congestion on a Geometric random graph.
\end{abstract}

Key-words: Wireless Ad-Hoc Networks, Wireless Sensor Networks, Random Graph Theory, Random Matrix Theory.

* alonso.silva@sophia.inria.fr

$\dagger$ Funded by Conicyt(Chile)/INRIA grant.

$\ddagger$ patricio.reyes@sophia.inria.fr

$\S$ merouane.debbah@supelec.fr

Centre de recherche INRIA Sophia Antipolis - Méditerranée

2004, route des Lucioles, BP 93, 06902 Sophia Antipolis Cedex

Téléphone : +334923877 77 — Télécopie : +33492387765 


\section{Congestion in Randomly Deployed Wireless Ad-Hoc and Sensor Networks}

Résumé : Congestion in wireless ad-hoc and sensor networks not only causes packet loss, and increases queueing delay, but also leads to unnecessary energy consumption. In a wireless ad-hoc and sensor network, two types of congestion can occur: node-level congestion, which is caused by buffer overflow in the node, or link-level congestion, when wireless channels are shared by several nodes and collisions occur when multiple active nodes try to seize the channel at the same time.

We study a measure of link-level congestion in a static wireless ad-hoc and sensor network randomly deployed over an area. The measure considered on this paper is the inverse of the greatest eigenvalue of the adjacency matrix of the random graph. This measure of congestion gives an approximation of the average quantity of wireless links of a certain length that a node have on the wireless ad-hoc and sensor network.

We review the results to find this measure of congestion in a Bernoulli random graph and we use tools from random graph theory and random matrix theory to extend this measure of congestion on a Geometric random graph.

Mots-clés : Wireless Ad-Hoc Networks, Wireless Sensor Networks, Random Graph Theory, Random Matrix Theory. 


\section{Introduction}

Wireless ad-hoc and sensor networks have gained much interest as inexpensive, energy-efficient, and miniaturized wireless devices are beginning to mature and take hold commercially. Wireless ad-hoc and sensor networks can be rapidly deployed as they do not require much existing infrastructure. Because of that, they are expected to find applications in many different settings, such as home appliance, disaster recovery, inventory tracking, battlefield surveillance, etc.

Congestion on this type of network is crucial as not only causes packet loss, and increases queueing delay, but also leads to unnecessary energy consumption, which causes lifetime reduction of the network. In wireless ad-hoc and sensor networks, extending the lifetime is important since all nodes contribute to collect the environment data and the early death of a node may lead to an incomplete monitoring. In a wireless adhoc and sensor network, two types of congestion can occur : node-level congestion, which is caused by buffer overflow in the node, or link-level congestion, when wireless channels are shared by several nodes and collisions occur when multiple active nodes try to seize the channel at the same time.

We will work on link-level congestion on randomly deployed static wireless ad-hoc and sensor networks. Wireless ad-hoc and sensor networks consist of nodes which share a common communication medium. On these networks, the signals intended for a receiver can cause interference at other receivers. The nodes on these networks cooperate in routing each other's data packets and communicate with each other over a wireless channel without any centralized control.

A wireless ad-hoc and sensor network can be seen as a graph $G$ with a finite sets of nodes, and links connecting pairs of nodes (its ends). We consider the boolean model of connectivity, i.e., two nodes are connected if the distance between them is inferior to a certain threshold (called range of connectivity), otherwise they are disconnected.

A path $P$ in a graph $G$ is a sequence $x_{0}, l_{1}, x_{1}, \ldots, l_{k}, x_{k}$ where each $x_{i}$ is a node, each $l_{i}$ is an link, and the ends of link $l_{i}$ are the nodes $x_{i-1}$ and $x_{i}$. The length of the path $P$ is $k$, i.e., the number of links on the path $P$ (see Fig. (1). The network is connected if each node is connected by means of a path to every other node in the network.

On this setting, Gupta and Kumar [1] derived the critical power at which a node in the network needs to transmit in order to ensure that the network is connected with probability one as the number of nodes in the network goes to infinity.

The main theorem of that paper is the following:

Theorem [Gupta-Kumar] [1] If n nodes are randomly located, uniformly i.i.d., in a disc of unit area and each node transmits at a power level so as to cover an area of $\pi r^{2}(n)=\frac{\log n+\gamma_{n}}{n}$, then the resulting network is asymptotically connected with probability one as $n \rightarrow+\infty$ if and only if $\gamma_{n} \rightarrow+\infty$.

On the rest of this paper we will assume that the network is connected, i.e., the range of connectivity is greater than the threshold given by Gupta-Kumar's Theorem.

Our goal is to provide for different randomly deployed wireless ad-hoc and sensor network topologies, the congestion of the network. In this paper we relate the notion of congestion to the number of paths of length $k$ and the spectral radius of the generated graph. Using tools of random graph theory and random matrix theory we are able to determine the number of paths of length $k$ there is on the network with $k$ large enough and to relate this quantity to the congestion of the network.

\section{Number of Paths of Length $k$ and Congestion on the Network}

In the following section, we analize the relationship between the number of paths of length $k$ in a wireless adhoc and sensor network and the link-level congestion over this network. In order to obtain this relationship we need to define some concepts of graphs and analyze the asymptotic behavior of the number of paths of length $k$.

RR $n^{\circ} 6854$ 


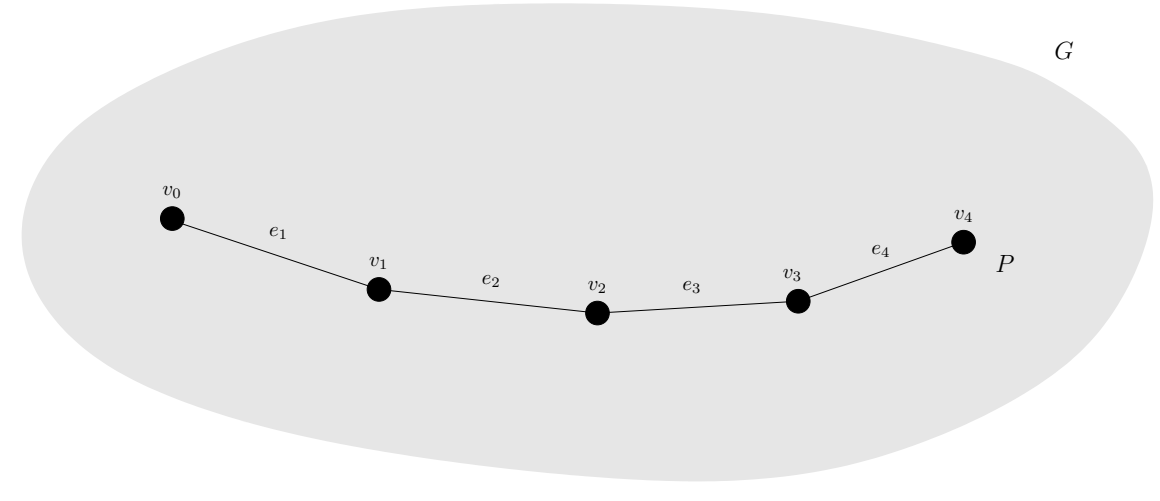

Figure 1: Path of length 4.

The ADJACENCY MATRIX of a graph $G$, denoted $A$, is the matrix with rows and columns labelled by graph vertices, defined as

$$
A_{i j}= \begin{cases}1 & \text { if } i \text { and } j \text { are connected by an link } \\ 0 & \text { otherwise. }\end{cases}
$$

The SPECTRAL RADIUS of a graph $G$, denoted $\lambda_{A}$, is the size of the largest eigenvalue of the adjacency matrix of the graph that can be written as

$$
\lambda_{A}=\sup _{x \in \mathbb{R}^{d} \backslash\{0\}} \frac{\langle x, A x\rangle}{\langle x, x\rangle} .
$$

Let $A$ denote the adjacency matrix of a graph $G$. Then $\left(A^{k}\right)_{i j}$ is the number of paths of length $k$ connecting the $i$-th and $j$-th vertices (proof by induction).

If we denote $\overrightarrow{1}$ the vector with all its components equal to $1 \mathrm{~s}$, then

$$
\overrightarrow{1}^{*} A^{k} \overrightarrow{1}=\sum_{i, j} A_{i j}^{k}
$$

is equal to the number of paths of length $k$ on the graph $G$.

The adjacency matrix is symmetric, then by spectral decomposition we have $A=\sum \lambda_{i} v_{i}$ where $v_{i}$ is the eigenvector of $A$ associated with the eigenvalue $\lambda_{i}$. Remember that as $A$ is a symmetric matrix, then the eigenvectors of $A$ associated with distinct eigenvalues are orthogonal.

The next theorem shows the importance of the spectral radius of a graph $G$.

Theorem [Perron-Frobenius] 2] Let $A$ be an irreducible matrix with non-negative entries and spectral radius $\lambda_{A}$. Then

1. $\lambda_{A}>0$,

2. $\lambda_{A}$ is an eigenvalue of $A$,

3. There is a unique eigenvector $v_{A}$ (up to a scale factor) with non-negative entries such that

$$
A v_{A}=\lambda_{A} v_{A}
$$

4. $\lambda_{A}$ is an algebraically simple eigenvalue of $A$. 
From this theorem, the following result holds:

$$
\begin{aligned}
\left(\overrightarrow{1}^{*} A^{k} \overrightarrow{1}\right)^{1 / k} & =\left(\sum a_{i} v_{i}^{*} A^{k} \sum a_{j} v_{j}\right)^{1 / k} \\
& =\left(\sum a_{i} v_{i}^{*} \sum a_{j} A^{k} v_{j}\right)^{1 / k} \\
& =\left(\sum a_{i} v_{i}^{*} \sum a_{j} \lambda_{j}^{k} v_{j}\right)^{1 / k} \\
& =\left(\sum\left|a_{i}\right|^{2} \lambda_{i}^{k}\right)^{1 / k} .
\end{aligned}
$$

This implies

$$
\lim _{k \rightarrow+\infty}\left(\overrightarrow{1}^{*} A^{k} \overrightarrow{1}\right)^{1 / k}=\lambda_{A}
$$

From this result we obtain that the number of paths of length $k$ in $G$ is approximately $\lambda_{A}^{k}$, for $k$ large enough.

Definition.- [Congestion Number] Given a graph $G$ we define the CONGESTION NumBER as the inverse of the spectral radius of the graph $\lambda_{A}^{-1}$.

The intuitive explanation to this definition is that while more paths of a fixed length we have in order to send information, we can split the information on these paths and coordinate it to arrive with the same number of hops at the receiver. This has the advantage of equalizing source-destination delays of packets that belong to the same class, which allows one to minimize the amount of packets that come out of sequence. This is desirable since in data transfers, out of order packets are misinterpreted to be lost which results not only in retransmissions but also in drop of systems throughput.

The following proposition give us another relationship between the spectral radius and on this case the degree of the nodes. The degree of a node in a graph is the number of links that connects to the node.

Proposition.- 3] Let $d_{\min }$ denote the minimum degree of $G$, let $\bar{d}$ be the average degree, and let $d_{\text {max }}$ be the maximum degree of $G$. For every graph $G$,

$$
\max \left\{\bar{d}, \sqrt{d_{\max }}\right\} \leq \lambda_{A} \leq d_{\max }
$$

\subsection{Discussion}

A fundamental question about any network is whether or not it is $\kappa$-connected, i.e., for each pair of different nodes there exists at least $\kappa$ link-disjoint paths in the graph connecting them of a fixed length that allow them to split their information and to send it through different paths. Additional requirements can be imposed, for instance the links can have small congestion.

We are conscious that the measure of congestion considered on this work has the limitation that the number of paths of fixed length are not necessarily link disjoint which would be an reasonable additional requirement. In that sense, a better measure would consider the possibility of splitting the information on independent paths without collision. However, for tractability reasons we consider this measure which is a good approximation and we can obtain explicit results.

\section{Analysis Tools}

In randomly deployed wireless ad-hoc and sensor networks the placement of the nodes and the links, which depend on the range of connectivity, are random. In order to derive the relation between congestion and spectral radius of a graph and to determine the spectral radius for different graphs, we use tools from random graph theory and random matrix theory. In the asymptotic case, it enables us to have a tractable expression of the number of paths of a fixed length. Similar tools have been used on [1] and [4] to analyze wireless ad-hoc and sensor networks.

RR $\mathrm{n}^{\circ} 6854$ 


\subsection{Random Graphs}

In this section we introduce some basic notions of random graphs.

Given $n$ nodes, $x_{1}, \ldots, x_{n}$, in $\mathbb{R}^{d}$ with $d=2$ or 3 , we denote by $G(n, r(n))$ the graph with set of nodes $\left\{x_{1}, \ldots, x_{n}\right\}$ and with links connecting all those nodes $x_{i}, x_{j}$, that satisfy $\left\|x_{i}-x_{j}\right\|<r(n)$ where $r(n)$ is the range of transmission and $\|\cdot\|$ is some norm in $\mathbb{R}^{d}$. We shall call $G(n, r(n))$ a GEOMETRIC GRAPH.

When the nodes are independent and identically distributed on $\mathcal{D}$ with a specific probability density function, the geometric graph $G(n, r(n))$ is called a GEOMETRIC RANDOM GRAPH.

In the following, the domain on which nodes are deployed is the $d$-dimensional cube $\mathcal{D}=[-1 / 2,1 / 2]^{d}$ where $d=2$ or 3 .

On this domain each node is deployed with uniform distribution, i.e.,

$$
f_{U}(x):= \begin{cases}1 & \text { if } x \in[-1 / 2,1 / 2]^{d} \\ 0 & \text { otherwise. }\end{cases}
$$

The most familiar random graph model, initiated by P. Erdös and A. Rényi [5] 6], consists of a graph with set of nodes $\left\{x_{1}, \ldots, x_{n}\right\}$, obtained by including some of the links of the complete graph, each link being included independently with probability $p$. The graph derived by the latter scheme is called a Bernoulli random graph and is denoted $G(n, p)$.

Bernoulli random graphs (also called Erdös-Renyi random graphs) have been intensively studied and many of their properties are by now well understood; see Bollobás [7] as a reference.

Bernoulli random graphs have the property of independence between the connectivity of different links, while for Geometric random graphs, if node $x_{i}$ is close to node $x_{j}$, and node $x_{j}$ is close to node $x_{k}$, then $x_{i}$ will be fairly close to $x_{k}$. In wireless ad-hoc and sensor networks, this property is more realistic than the independence of links as in the Bernoulli random graphs.

Examples of Bernoulli random graphs $G(n, p)$ for different $p$ 's and of Geometric random graphs $G(n, r)$ for different $r$ 's can be found in figures 2 and 3 respectively.

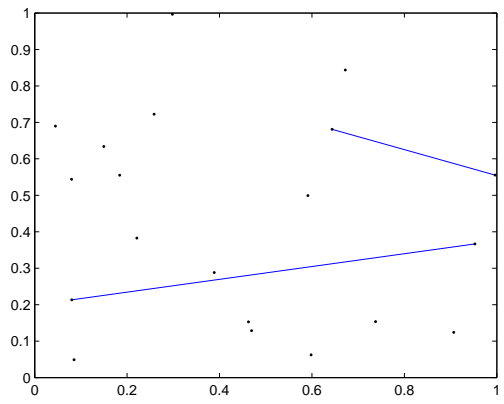

(a) Bernoulli random graph $G(n, p)$ with $n=20$ and $p=0.01$

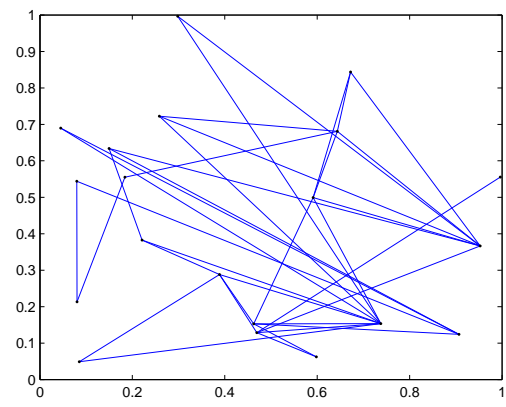

(b) Bernoulli random graph $G(n, p)$ with $n=20$ and $p=0.08$

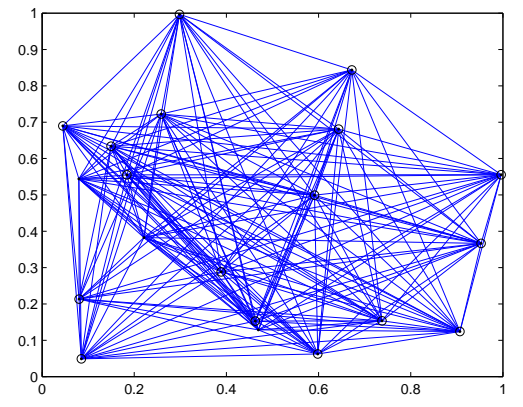

(c) Bernoulli random graph $G(n, p)$ with $n=20$ and $p=0.8$

Figure 2: Bernoulli random graphs

From the figures 4(a) and 4(b) done by simulation, we see that the convergence of the $k$-th root of the numbers of paths of length $k$ converges very fast to the spectral radius with respect to $k$ on these two settings.

\subsection{Random Matrix Theory}

The main application of random matrix theory lies on the derivation of asymptotic results for large random matrices. In many practical cases, the eigenvalue distribution of large random hermitian matrices converges 


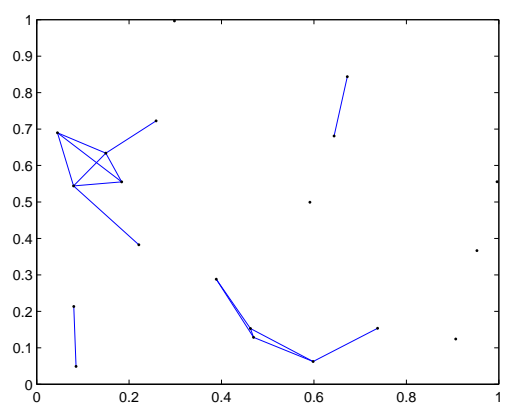

(a) Geometric random graph $G(n, r)$ with $n=20$ and $r=1 / 6$

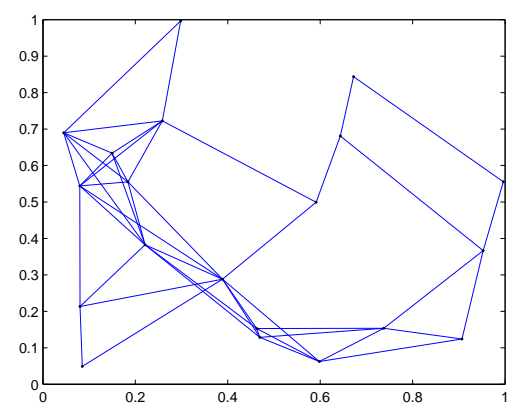

(b) Geometric random graph $G(n, r)$ with $n=20$ and $r=1 / 3$

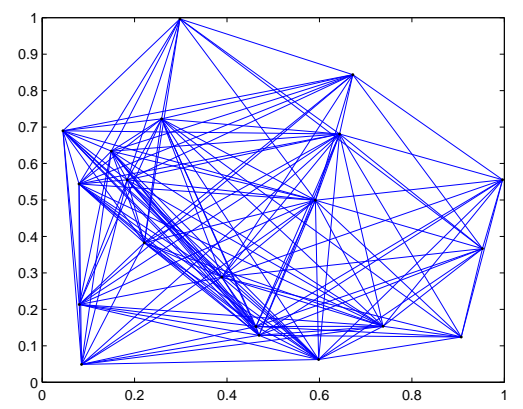

(c) Geometric random graph $G(n, r)$ with $r=2 / 3$

Figure 3: Geometric random graphs

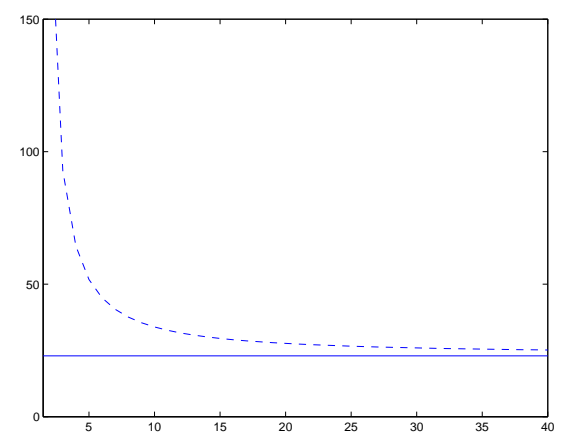

(a) In a Geometric random graph.

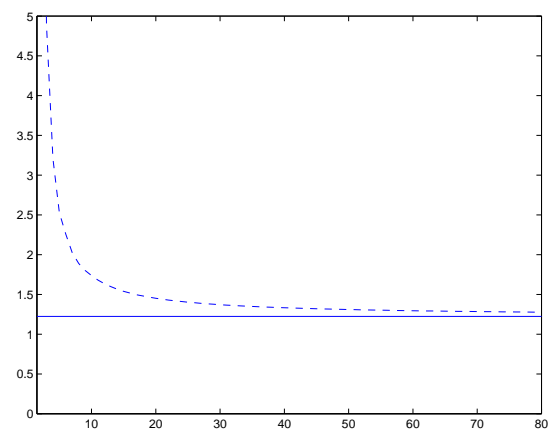

(b) In a Bernoulli random graph.

Figure 4: Convergence of the $k$-th root of the number of paths of length $k$ (depicted as a dashed curve) to the spectral radius of the graph $\lambda_{A}$ (solid curve) with respect to $k$. 
to a definite probability distribution, called empirical distribution or density of states. In particular, we can also find the value or bounds of the largest or smallest eigenvalues of large random hermitian matrices.

In this work we will use random matrix theory to derive the spectral radius of a Geometric random graph.

Definition.- An Euclidean random matrix is an $n \times n$ matrix, $A$, whose entries are a function of the positions of $n$ random points in a compact set $\mathcal{D}$ of $\mathbb{R}^{d}$.

More precisely, if $n$ nodes, $x_{1}, \ldots, x_{n}$, are located randomly, uniformly i.i.d., in a square of unit area $\mathcal{D}$ and the matrix $A$ is defined as

$$
A:=\left(F\left(x_{i}-x_{j}\right)\right)_{1 \leq i \leq j \leq n}
$$

where $F$ is a measurable mapping from $\mathbb{R}^{d}$ to $\mathbb{C}$. Then $A$ is an Euclidean random matrix.

We consider the boolean model of connectivity, i.e., two nodes are connected if the distance between them is inferior to a certain threshold and otherwise they are disconnected. Therefore, if $n$ nodes are located randomly, uniformly i.i.d., in a square of unit area and each node transmits at a power in order to cover an area of $r(n)$, then the adjacency matrix of this random graph is given by

$$
A_{i j}=1_{\left\{\left\|x_{i}-x_{j}\right\| \leq r(n)\right\}}
$$

where

$$
1_{\left\{\left\|x_{i}-x_{j}\right\| \leq r(n)\right\}}= \begin{cases}1 & \text { if }\left\|x_{i}-x_{j}\right\| \leq r(n) \\ 0 & \text { otherwise. }\end{cases}
$$

We would like to determine for this adjacency matrix the maximum eigenvalue or spectral radius and relate it to the congestion on the network.

In order to determine the congestion number, we explicit recent results of Bordenave on Geometric random graphs. Following the paper of Bordenave [], we assume that the discrete Fourier transform of $F$ is defined for all $k \in \mathbb{Z}^{d}$ where

$$
\hat{F}(k)=\int_{\mathcal{D}} F(x) e^{-2 \pi i k \cdot x} \mathrm{~d} x
$$

We assume that almost everywhere (a.e.) and at 0, the Fourier series of $F$ exists and

$$
F(x)=\sum_{k \in \mathbb{Z}^{d}} \hat{F}(k) e^{2 \pi i k \cdot x}
$$

A sufficient condition for the existence of the Fourier series of $F$ (a.e.) is that

$$
\sum_{k \in \mathbb{Z}^{d}}|\hat{F}(k)|<+\infty
$$

and $F$ to be continuous at zero.

Let's define $A_{n}=A / n$ and

$$
\mu_{n}=\sum_{i=1}^{n} \delta_{\lambda_{i}(n) / n}
$$

where $\left\{\lambda_{i}(n)\right\}_{1 \leq i \leq n}$ is the set of eigenvalues of $\mathrm{A}$ and $\delta$ is the dirac function. Notice that $\left\{\lambda_{i}(n) / n\right\}_{1 \leq i \leq n}$ is the set of eigenvalues of $A_{n}$.

Let's define the measure

$$
\mu=\sum_{k \in \mathbb{Z}} \delta_{\hat{F}(k)}
$$

The following theorem gives us the convergence of the empirical distribution or density of states to a nonrandom distribution characterized by the Fourier transform of the function $F$.

Theorem [Bordenave] 8 ] In the previous setting

$$
\lim _{n \rightarrow+\infty} \mu_{n}(K)=\mu(K) \quad \text { a.e. }
$$


for all Borel sets $K$ with $\mu(\partial K)=0$ and $0 \notin \bar{K}$.

The following corollary gives us a formula to compute the spectral radius of a graph.

Corollary [8] The convergence of the spectral radius of $A_{n}$, almost surely, is given by

$$
\lim _{n \rightarrow+\infty} \max _{1 \leq i \leq n} \frac{\left|\lambda_{i}(n)\right|}{n}=\max _{k \in \mathbb{Z}^{d}}|\hat{F}(k)| .
$$

Having this corollary in mind we can compute for different norms the spectral radius of a graph.

The following norms will be considered:

$$
\begin{aligned}
\|x\|_{\infty} & :=\max \left\{\left|x_{1}\right|, \ldots,\left|x_{n}\right|\right\} \text { (Infinity norm) } \\
\|x\|_{p} & :=\left(\sum_{i=1}^{n}\left|x_{i}\right|^{p}\right)^{1 / p} \quad(p \text {-norm) } \quad \forall p>1 \\
\|x\|_{1} & :=\sum_{i=1}^{n}\left|x_{i}\right| \text { (Manhattan norm) }
\end{aligned}
$$

Note that with the infinity norm case we obtain a closed form expression given by

$$
F(x)=1_{\left\{\max _{1 \leq i \leq d}\left|x_{i}\right| \leq r\right\}}(x),
$$

for which its discrete Fourier transform writes as

$$
\hat{F}(k)=r^{d} \prod_{i=1}^{d} \frac{\sin \left(2 \pi k_{i} r\right)}{2 \pi k_{i} r}
$$

where $k=\left(k_{1}, \ldots, k_{d}\right) \in \mathbb{Z}$.

Then for the infinity norm the spectral radius is given by $r^{d}$.

The figures 6(a) and 6(b) give us the asymptotic convergence of the spectral radius of the adjacency matrix to the maximum of the Fourier transform over the $d$-dimensional integer lattice with respect to the number of nodes on the network.

There is a similar result on the spectral radius of a Bernoulli random graph $A$ that we put for completeness.

Theorem [Füredi-Komlós] [] Let $a_{i j}, i \geq j$, be independent (not necessarily identically distributed) random variables bounded with a common bound $K$. Assume that for $i>j$, the $a_{i j}$ have a common expectation $\mu$ and variance $\sigma^{2}$, further that $\mathbb{E}\left(a_{i i}\right)=\nu$. Define $a_{i j}$ for $i<j$ by $a_{i j}=a_{j i}$ (the numbers $K, \mu, \sigma^{2}, \nu$ will be kept fixed as $n$ will tend to infinity).

If $\mu>0$ then the distribution of the largest eigenvalue of the random symmetric matrix $A=\left(a_{i j}\right)$ can be approximated in order $1 / \sqrt{n}$ by a normal distribution of expectation

$$
(n-1) \mu+\nu+\sigma^{2} / \mu
$$

and variance $2 \sigma^{2}$.

The result of this theorem stems from the analysis of the largest eigenvalue of non-zero mean random matrices with independent entries.

From this theorem in our case the constants are $K=1, \mu=p, \nu=0$ and $\sigma^{2}=p(1-p)$ and then the expected spectral radius of a Bernoulli random graph is $(n-1) p+(1-p)$. 


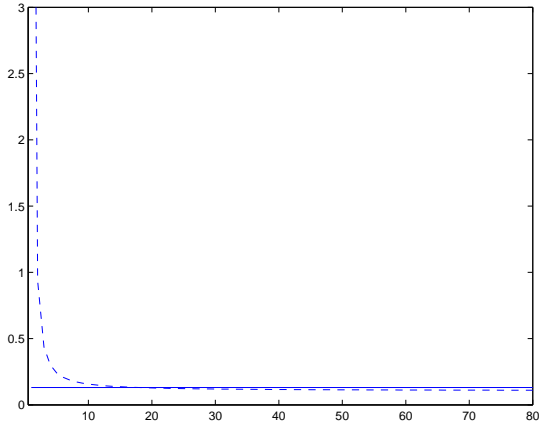

(a) In a Geometric random graph.

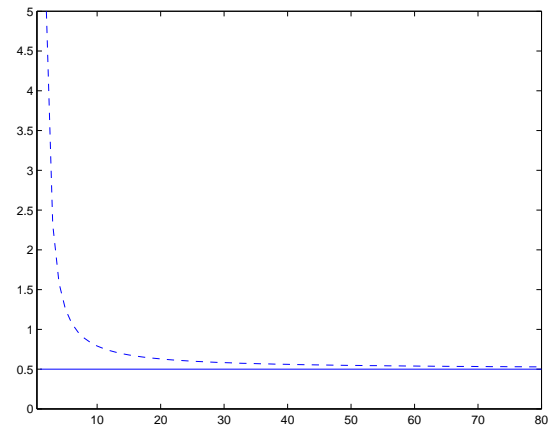

(b) In a Bernoulli random graph.

Figure 5: Convergence of the $k$-th root of the number of paths of length $k$ (depicted as a dashed curve) to the estimation of the spectral radius (solid curve) given by Bordenave's Theorem and Füredi-Komlós' Theorem, respectively, with respect to $k$.

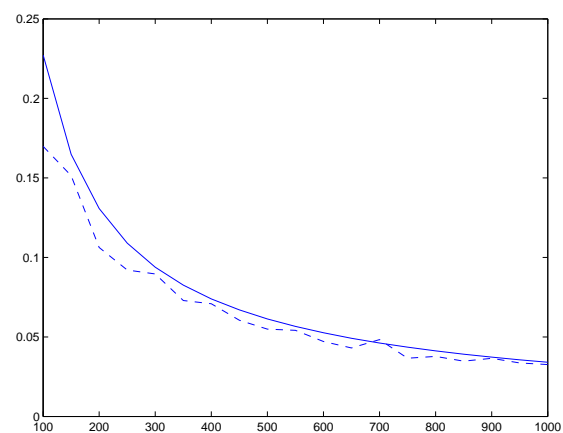

(a) In a Geometric random graph.

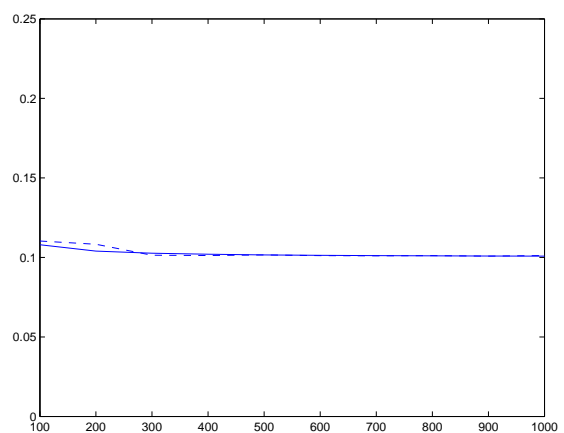

(b) In a Bernoulli random graph.

Figure 6: Convergence of the largest eigenvalue (dashed curve) to the asymptotic approximation (solid curve) given by Bordenave's Theorem and Füredi-Komlós' Theorem, respectively. 


\section{Conclusions and Future Work}

In this contribution, we have provided a model to deal with congestion of randomly deployed wireless nodes. For various cases of random graphs (Bernoulli random graphs and Geometric random graphs), we have provided, in the case of large networks, the congestion number which is linked to the number of connected paths of a given length. Quite remarkably, the mean congestion number can be explicitly derived using asymptotic results of random matrix theory and the results holds even for a not so large number of nodes. Further studies will focus on providing central limit theorems on the congestion number in order to have a better assessment of the quality of service in the network. Other realistic models (beside the boolean model for connectivity) will also also studied in combination with other random distribution of the nodes.

\section{Acknowledgement}

The authors want to thank Dr. Charles Bordenave for helpful discussions.

\section{References}

[1] P. Gupta and P. R. Kumar, "Critical power for asymptotic connectivity in wireless networks," Stochastic Analysis, Control, Optimization and Applications: A volume in honor of W.H. Fleming, (eds W. M. McEneany, G. Yin, and Q. Zhang), Birkäuser, Boston, pp. 547-566, 1998.

[2] R. A. Horn and Ch. R. Johnson, "Matrix Analysis," pp. 508, Cambridge University Press, 1990.

[3] L. Lovász, "Eigenvalues of graphs."

[4] F. Chung and L. Lu, "Complex Graphs and Networks," CBMS Regional Conference Series in Mathematics, vol. 107, American Mathematical Society, 2006.

[5] P. Erdös, Graph Theory and Probability, Canad. J. Math, vol. 11, pp 34-38,1959.

[6] P. Erdös and A. Rényi, "On Random Graphs", Publ. Math. Debrecen, vol. 6, pp. 290-297, 1959.

[7] B. Bollobás, "Random Graphs", Academic Press, London, 1985.

[8] C. Bordenave, "Eigenvalues of Euclidean Random Matrices", Random Struct. Algorithms, vol. 33, pp. $515-532,2008$.

[9] Z. Füredi and J. Komlós, "The eigenvalues of random symmetric matrices," Combinatorica, vol. 1, no. 3 , pp. 233-241, 1981.

[10] M. Penrose, "Random Geometric Graphs," Oxford University press, 2003.

[11] F. Chung, "Spectral Graph Theory", Published for the Conference Board of the Mathematical Sciences, Washington, DC, 1997.

RR $n^{\circ} 6854$ 


\section{Contents}

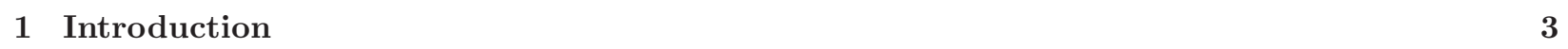

2 Number of Paths of Length $k$ and Congestion on the Network 3

2.1 Discussion . . . . . . . . . . . . . . . . . . . . . . . . . . . . . 5

3 Analysis Tools $\quad 5$

3.1 Random Graphs . . . . . . . . . . . . . . . . . . . . . . 6

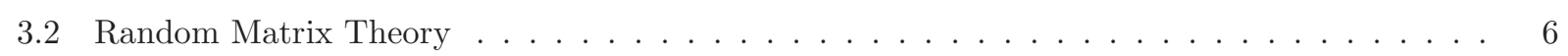

4 Conclusions and Future Work $\quad 10$ 


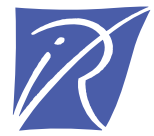

Centre de recherche INRIA Sophia Antipolis - Méditerranée 2004, route des Lucioles - BP 93 - 06902 Sophia Antipolis Cedex (France)

Centre de recherche INRIA Bordeaux - Sud Ouest : Domaine Universitaire - 351, cours de la Libération - 33405 Talence Cedex Centre de recherche INRIA Grenoble - Rhône-Alpes : 655, avenue de l'Europe - 38334 Montbonnot Saint-Ismier Centre de recherche INRIA Lille - Nord Europe : Parc Scientifique de la Haute Borne - 40, avenue Halley - 59650 Villeneuve d'Ascq Centre de recherche INRIA Nancy - Grand Est : LORIA, Technopôle de Nancy-Brabois - Campus scientifique 615, rue du Jardin Botanique - BP 101 - 54602 Villers-lès-Nancy Cedex

Centre de recherche INRIA Paris - Rocquencourt : Domaine de Voluceau - Rocquencourt - BP 105 - 78153 Le Chesnay Cedex

Centre de recherche INRIA Rennes - Bretagne Atlantique : IRISA, Campus universitaire de Beaulieu - 35042 Rennes Cedex

Centre de recherche INRIA Saclay - Île-de-France : Parc Orsay Université - ZAC des Vignes : 4, rue Jacques Monod - 91893 Orsay Cedex

INRIA - Domaine de Voluceau - Rocquencourt, BP 105 - 78153 Le Chesnay Cedex (France) http://www.inria.fr

ISSN 0249-6399 